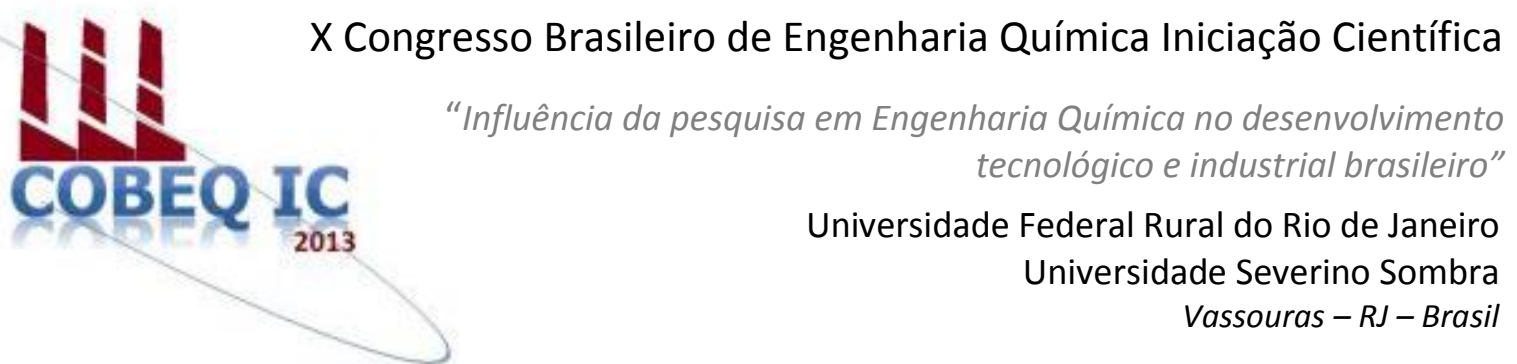

\title{
INFLUÊNCIA DO SUBSTRATO NA DETERMINAÇÃO DA ATIVIDADE DA LIPASE DE Yarrowia lipolytica DURANTE A IMOBILIZAÇÃO POR ADSORÇÃO EM HIDRÓXIDO DUPLO LAMELAR (HDL)
}

\author{
RIOS $^{* 1}$, N.S.; DE MATOS ${ }^{2}$, L.J.B.L.; DA SILVA JÚNIOR ${ }^{3}$, I.J.; AMARAL ${ }^{4}$, P.F.F.; \\ COELHO $^{5}$, M.A.Z.; GONÇALVES ${ }^{6}$, L.R.B \\ ${ }^{1}$ Aluna do DEQ/UFC $\quad{ }^{2}$ Doutorando do DEQ/UFC ${ }^{36}$ Professor (a) do DEQ/UFC \\ ${ }^{45}$ Professora do DEB/UFRJ \\ Departamento de Engenharia Química - Universidade Federal do Ceará \\ Endereço-Campus do Pici, Bloco 709 - CEP 60455-760 \\ email: lrg@ufc.br
}

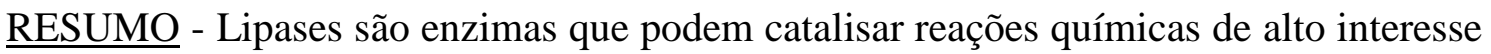
industrial, como a produção de biodiesel. As enzimas solúveis são muito sensíveis a alterações de temperatura e $\mathrm{pH}$, o que dificulta sua aplicação na indústria. Frente a esta dificuldade, estuda-se a imobilização de enzimas em suportes sólidos. O método de imobilização utilizado foi adsorção física da lipase no suporte, sendo as interações enzima/suporte por meio de ligações fracas, não covalentes. Nesse contexto, foram realizados experimentos para determinar a influência da afinidade enzima/substrato, nas mesmas condições de operação, na determinação dos parâmetros de imobilização. Os substratos testados para a determinação da atividade da enzima foram o p-nitrofenil laurato (pNFL) e azeite de oliva, utilizando uma emulsão preparada com goma arábica. Um estudo a diferentes temperaturas $\left(4^{\circ} \mathrm{C}, 10^{\circ} \mathrm{C}, 25^{\circ} \mathrm{C}, 30^{\circ} \mathrm{C}, 37^{\circ} \mathrm{C}\right)$ comparando os substratos foi realizado. Os melhores resultados foram observados na temperatura de 10 ${ }^{\circ} \mathrm{C}$, para o substrato azeite de oliva (Atividade da enzima imobilizada: $31,43 \mathrm{U} / \mathrm{g}$ e Atividade recuperada: $37,41 \%$ ). Os parâmetros de imobilização determinado a partir do substrato pNFL foram menores (Atividade da enzima imobilizada: 6,42 U/g e Atividade recuperada: $7,03 \%$ ), mostrando que a lipase tem uma maior afinidade pelo substrato azeite de oliva.
\end{abstract}

Palavras chave: lipase, p-nitrofenil laurato, azeite de oliva.

\section{INTRODUÇÃO}

As enzimas atuam como biocatalisadores acelerando reações químicas. Estas possuem uma alta eficiência catalítica, pois a enzima pode acelerar uma reação na ordem de $10^{8}$ a
$10^{12}$ vezes, mantendo uma alta seletividade (Castro et al., 2004). As lipases são enzimas que catalisam reações de elevado interesse industrial, síntese de ésteres na produção do biodiesel. 
As enzimas solúveis são muito sensíveis a alterações de temperatura e $\mathrm{pH}$, o que dificulta sua aplicação na indústria. Neste contexto, estuda-se a imobilização da lipase de Yarrowia lipolytica em suportes sólidos (Cunha et al., 2007; Yan et al., 2013), empregando alguns métodos de imobilização, tais como adsorção física, ligação covalente, entre outros.

Neste trabalho, o método de imobilização estudado foi a adsorção física da lipase de Yarrowia lipolytica em Hidróxido Duplo Lamelar (HDL). O método da adsorção física é de baixo custo, no entanto, as interações enzima/suporte são de natureza fraca, como interações hidrofóbicas, forças de Van der Waals, entre outras (Brígida et al., 2008).

Neste estudo, a influência da afinidade enzima/substrato, nas mesmas condições de operação, na determinação dos parâmetros de imobilização foi investigada. Os substratos utilizados para a determinação da atividade da enzima foram o p-nitrofenil laurato (pNFL) e azeite de oliva, utilizando uma a emulsão preparada com goma arábica.

\section{MATERIAIS E MÉTODOS}

\section{Preparação do Suporte Hidróxido Duplo Lamelar (HDL)}

O suporte HDL foi sintetizado pelo método de co-precipitação a pH variável utilizando uma solução aquosa contendo sais de cátions $\left(\mathrm{Mg}\left(\mathrm{NO}_{3}\right)_{3} \cdot 6 \mathrm{H}_{2} \mathrm{O}\right.$ e $\left.\mathrm{Al}\left(\mathrm{NO}_{3}\right)_{3} \cdot 9 \mathrm{H}_{2} \mathrm{O}\right)$ em uma razão molar $\mathrm{Mg} / \mathrm{Al}=3: 1$. A síntese do material foi de acordo com a metodologia apresentada por Aguiar et al., 2013.

\section{Imobilização Enzimática}

A lipase de Yarrowia lipolytica foi imobilizada no suporte HDL nas temperaturas $\left(4{ }^{\circ} \mathrm{C}, 10^{\circ} \mathrm{C}, 25^{\circ} \mathrm{C}, 30^{\circ} \mathrm{C}, 37^{\circ} \mathrm{C}\right)$. A atividade oferecida inicial de enzima foi de $95 \mathrm{U} / \mathrm{g}$. A imobilização foi realizada em presença do tampão fosfato de sódio $25 \mathrm{mM}, \mathrm{pH} 7$. A proporção de imobilização utilizada foi de $1 / 20(\mathrm{~m} / \mathrm{v})$.

\section{Determinação da atividade hidrolítica da lipase em p-nitrofenil laurato (pNFL)}

A atividade da lipase foi determinada através da hidrólise do substrato p-nitrofenil laurato (pNFL), liberando o p-nitrofenol como produto. Dessa forma, foi possível avaliar a atividade da enzima solúvel, do sobrenadante após o processo de imobilização e da enzima imobilizada ( $\left.\mathrm{At}_{\mathrm{D}}\right)$. (Rios et al., 2013). A atividade é calculada pela equação 1 :

$$
A t=\frac{\text { C. F } . \text { Vr }}{\text { Ve ou Md }}
$$

onde At é a atividade enzimática $(\mathrm{U} / \mathrm{mL}$ ou U/g), C é a taxa de consumo do pNFL ( $\mu \mathrm{mol} / \mathrm{mL} \cdot \mathrm{min}), \mathrm{F}$ é o fator de diluição da solução enzimática, Vr é o volume do reator, Ve é o volume de solução enzimática e Md a massa de derivado. Para a atividade da enzima imobilizada, foram realizados ensaios em um reator em bancada com $30 \mathrm{~mL}$ de pNFL em contato com $0,1 \mathrm{~g}$ da lipase adsorvida no suporte. $\mathrm{O}$ sistema foi mantido sob agitação a $37^{\circ} \mathrm{C}$ e a reação de hidrólise foi monitorada durante 20 minutos.

\section{Determinação da atividade hidrolítica no substrato azeite de oliva emulsionado com goma arábica}

A emulsão foi preparada adicionando-se $13,75 \mathrm{~g}$ de azeite de oliva e 41,25 g de solução de goma arábica (7 \% $\mathrm{m} / \mathrm{v}$ em água). Posteriormente, $55 \mathrm{~mL}$ de tampão fostato de sódio $100 \mathrm{mM}, \mathrm{pH}=7$ foram adicionados. A mistura foi vigorosamente agitada a 13000 rpm. A metodologia foi modificada de De Aguiar et al. (2010). Os frascos foram incubados a $37{ }^{\circ} \mathrm{C}, 160 \mathrm{rpm}$ por 5 minutos. Após o período de incubação, a reação foi monitorada por 20 minutos. Os ácidos graxos liberados foram mensurados titulando-se $\mathrm{KOH}$ 0,025M devidamente padronizado.

A atividade da enzima livre em $\mathrm{U} / \mathrm{mL}$ e a atividade da enzima imobilizada em $\mathrm{U} / \mathrm{g}$ foram mensuradas pela equação 2 :

$$
A t=\frac{(V a-V b) \cdot M \cdot F \cdot 1000}{t \cdot V e}
$$

Em que Va é o volume de $\mathrm{KOH}$ titulado, $\mathrm{Vb}$ é o volume do branco, $\mathrm{M}$ é a molaridade real do $\mathrm{KOH}$, F representa o Fator de Diluição; Ve é o volume de solução enzimática e t é o 
tempo de reação. Para a determinação da atividade da enzima imobilizada utiliza-se a equação (II), substituindo o volume da enzima pela massa do derivado.

\section{Parâmetros de Imobilização}

Os parâmetros de imobilização foram mensurados de acordo com Silva et al., 2012. $\mathrm{O}$ rendimento de imobilização $(\mathrm{R})$ é a quantidade de enzima teoricamente imobilizada. A atividade teórica pode ser calculada utilizando a quantidade de enzima oferecida inicialmente por grama de suporte e o rendimento de imobilização (Silva et al., 2012). A atividade recuperada é mensurada utilizando a atividade da enzima imobilizada. As equações são apresentadas abaixo:

Atividade Teórica $\left(\mathrm{At}_{\mathrm{T}}\right)=\mathrm{R}$. CO

$$
\text { Atividade Recuperada }\left(A t_{R}\right)=\frac{A t_{D}}{\mathrm{At}_{\mathrm{T}}} \cdot 100
$$

\section{RESULTADOS E DISCUSSÃO}

Realizou-se a adsorção da lipase de Yarrowia lipolytica no suporte HDL em várias temperaturas, a fim de determinar a condição ideal para o processo. Paralelamente, a atividade hidrolítica da enzima foi mensurada em dois substratos, determinando-se os parâmetros de imobilização.

Os substratos estudados foram o pnitrofenil laurato (pNFL) e o azeite de oliva em uma emulsão com goma arábica. Os resultados são apresentados nas tabelas 1 e 2, abaixo.

Tabela 1 - Parâmetros de imobilização da lipase medidos com o substrato pNFL.

\begin{tabular}{|c|c|c|c|c|c|}
\hline \multicolumn{1}{|c}{$\left(^{\circ} \mathbf{C}\right)$} & $4^{\circ} \mathbf{C}$ & $\mathbf{1 0}^{\circ} \mathbf{C}$ & $\mathbf{2 5}^{\circ} \mathbf{C}$ & $\mathbf{3 0}^{\circ} \mathbf{C}$ & $3^{\circ} \mathbf{C}$ \\
\hline $\mathrm{R}(\%)$ & 86,05 & 96,09 & 80,71 & 99,72 & 96,41 \\
\hline $\begin{array}{c}\mathrm{At}_{\mathrm{T}} \\
(\mathrm{U} / \mathrm{g})\end{array}$ & 81,75 & 91,29 & 76,68 & 94,74 & 91,33 \\
\hline $\begin{array}{c}\mathrm{At}_{\mathrm{D}} \\
(\mathrm{U} / \mathrm{g})\end{array}$ & 4,69 & 6,42 & 4,34 & 0,00 & 0,00 \\
\hline $\begin{array}{c}\mathrm{At}_{\mathrm{R}} \\
(\%)\end{array}$ & 5,75 & 7,03 & 5,73 & 0,00 & 0,00 \\
\hline
\end{tabular}

Observando os resultados da tabela 1, pode-se afirmar que a melhor temperatura de imobilização foi $10^{\circ} \mathrm{C}$, pois obtiveram-se altos rendimentos de imobilização e a enzima permaneceu mais ativa após o processo de adsorção (Atividade do derivado: 6,42 U/g).

Tabela 2 - Parâmetros de imobilização da lipase medidos com o substrato azeite de

\begin{tabular}{|c|c|c|c|c|c|}
\hline $\mathrm{T}\left({ }^{\circ} \mathrm{C}\right)$ & $4^{\circ} \mathrm{C}$ & $1^{\circ} \mathrm{C}$ & $25^{\circ} \mathrm{C}$ & $30^{\circ} \mathrm{C}$ & $37^{\circ} \mathrm{C}$ \\
\hline $\mathrm{R}(\%)$ & 74,35 & 88,43 & 100 & 100 & 99,01 \\
\hline $\begin{array}{c}\mathrm{At}_{\mathrm{T}} \\
(\mathrm{U} / \mathrm{g})\end{array}$ & 70,73 & 84,02 & 95 & 95 & 94,09 \\
\hline $\begin{array}{c}\mathrm{At}_{\mathrm{D}} \\
(\mathrm{U} / \mathrm{g})\end{array}$ & 13,12 & 31,43 & 14,38 & 5,47 & 2,49 \\
\hline $\begin{array}{l}\mathrm{At}_{\mathrm{R}} \\
(\%)\end{array}$ & 18,58 & 37,41 & 15,14 & 5,76 & 2,65 \\
\hline
\end{tabular}
oliva em uma emulsão com goma arábica.

Analisando os resultados da tabela 2, pode-se afirmar que a melhor temperatura de imobilização também foi $10{ }^{\circ} \mathrm{C}$. Isso mostra que a variação de substratos não altera o comportamento enzimático. No entanto, a variação de substratos altera a expressão da atividade hidrolítica da enzima de acordo com o grau de afinidade da lipase pelo substrato.

A afinidade de uma lipase em um determinado substrato está relacionada diretamente com a interação deste com os resíduos de aminoácidos presentes no sítio catalítico da lipase bem como com o tamanho e formato do mesmo (BRÍGIDA, 2010).

Comparando as tabela 1 e 2, pode-se afirmar que a lipase de Yarrowia lipolytica possui maior afinidade pelo substrato azeite de oliva em emulsão com goma arábica. Isso é explicado pelas maiores atividades da enzima imobilizada $\left(\mathrm{At}_{\mathrm{D}}\right)$ e atividades recuperadas $\left(\mathrm{At}_{\mathrm{R}}\right)$.

\section{CONCLUSÃO}

Constatou-se que a melhor temperatura de imobilização da lipase de Yarrowia lipolytica foi de $10^{\circ} \mathrm{C}$. O substrato azeite de oliva emulsionado com goma arábica é mais apropriado para medir os parâmetros de imobilização, pois foram obtidos maiores valores de atividades do derivado e recuperada. Isso indica que a enzima estudada tem maior afinidade por este substrato. 


\section{REFERÊNCIAS}

AGUIAR, J. E.; BEZERRA, B. T. C.; BRAGA, B. D. M.; LIMA, P. D. D. S.; NOGUEIRA, R. E. F. Q.; DE LUCENA, S. M. P.; DA SILVA, I. J. (2013) "Adsorption of Anionic and Cationic Dyes from Aqueous Solution on NonCalcined Mg-Al Layered Double Hydroxide: Experimental and Theoretical Study." Separation Science and Technology, v. 48, n. 15, p. 2307-2316.

BRIGIDA, A. I.; PINHEIRO, A. D.; FERREIRA, A. L.; GONCALVES, L. R. (2008) "Immobilization of Candida antarctica lipase B by adsorption to green coconut fiber". Appl Biochem Biotechnol, v. 146, n. 1-3, p. 173-87.

BRIGIDA, A. I. S. (2010), Imobilização de lipases utilizando fibra da casca de coco verde como suporte para aplicações industriais. Tese. Escola de Química. Universidade Federal do Rio de Janeiro. Rio de Janeiro. 197p.

CASTRO, H.F.; MENDES, A.A.; DOS SANTOS, J.C.; DE AGUIAR, C.L. (2004) "Modificação de óleos e gorduras por biotransformação" Quim. Nova. v. 27, n.1, p. 146-156.

CUNHA, V. R. R.; DA C. FERREIRA, A. M.; CONSTANTINO, V. R. L.; TRONTO, J.; VALIM, J. B. (2010) "Layered double hydroxides: Inorganic nanoparticles for storage and release of species of biological and therapeutic interest". Química Nova, v. 33, n. 1, p. 159-171.

DE AGUIAR, R. O.; MONDARDO, R. M.; AGNES, E. J.; DE CASTRO, H. F.; PEREIRA, E. B. (2010) "Evaluation and comparison of the efficiency of detention in chitosan pancreatic lipase for production of fatty acids in flasks under shaking." Acta Scientiarum. Technology, v. 32, n. 1, p. 15-19.

RIOS, N. S.; PINHEIRO, B. B.; MATOS, L. J. B. L.; SILVA JR., I. J.; AMARAL, P.F.F.; COELHO, M. A. Z.; GONÇALVES, L.R.B. (2013) "Imobilização da lipase de Yarrowia lipolytica por adsorção: influência do suporte e do tempo de contato" Anais de XIX Simpósio Nacional de Bioprocessos - SINAFERM 2013, 2013, Foz do Iguaçu - PR, v. 1. p. 1-4.

SILVA, J. A.; MACEDO, G. P.; RODRIGUES, D. S.; GIORDANO, R. L. C.; GONÇALVES, L. R. B. (2012) "Immobilization of Candida antarctica lipase B by covalent attachment on chitosan-based hydrogels using different support activation strategies" Biochemical Engineering Journal, v. 60, p. 16-24.

YAN, Y.; ZHANG, X.; CHEN, D. (2013) "Enhanced catalysis of Yarrowia lipolytica lipase LIP2 immobilized on macroporous resin and its application in enrichment of polyunsaturated fatty acids" Bioresour Technol, v. 131, p. 179-87.

\section{AGRADECIMENTOS}

Os autores agradecem o PRH-31 - ANP, PETROBRAS, CAPES e CNPQ pelo auxílio financeiro concedido para a realização do trabalho. 